\title{
Sarcoidosis: una serie de casos del nororiente colombiano
}

\author{
Yeison Santamaria-Alza, $\bowtie$ Javier Enrique Fajardo Rivero
}

Universidad Industrial de Santander - Hospital Universitario de Santander, Bucaramanga, Colombia.

Trabajo recibido: 12-IX-2016; aceptado: 28-X-2016

\begin{abstract}
RESUMEN. Antecedentes: La sarcoidosis es una enfermedad sistémica de origen no establecido que afecta principalmente a adultos jóvenes. El sistema afectado principalmente es el respiratorio. El objetivo del presente trabajo es mostrar la experiencia de un centro de referencia del nororiente colombiano para la generación de hipótesis y futuros trabajos de investigación en el tema. Métodos: Se incluyeron todos los pacientes con diagnóstico histopatológico de sarcoidosis del Servicio de Consulta Externa de Neumología del Hospital Universitario de Santander. Se realizó análisis descriptivo de los sujetos. Resultados: En total se incluyeron 8 pacientes, $50 \%$ de ellos fueron hombres, edad promedio 39.75 años. El síntoma con más frecuencia presentado en los pacientes con sarcoidosis fue la disnea. El compromiso pulmonar se evidenció en la mitad de los pacientes con predominio bilateral y de lóbulos superiores. El estado más prevalente de la sarcoidosis fue el estado I en el 50\% de los casos. Conclusión: La sarcoidosis, aunque es una enfermedad infrecuente, representa un reto en el diagnóstico y tratamiento para el médico. En la serie presentada se encuentran datos compatibles con los reportados en otras latitudes.
\end{abstract}

Palabras clave: Sarcoidosis, sarcoidosis pulmonar, enfermedades pulmonares.

ABSTRACT. Background: Sarcoidosis is a systemic disease of unestablished origin, that mainly affects the respiratory system as young adults. This paper's objective is to show the experience of the Reference Center northeastern Colombia, for hypothesis generation and future research on the topic. Methods: All patients with histopathological diagnosis of sarcoidosis, attended in pulmonology service as out-patients at Santander's University Hospital. Descriptive analysis was performed to the subjects. Results: 8 subjects were included, $50 \%$ of subjects were male, mean age 39.75 years. The most often occurred symptom in patients with sarcoidosis was dyspnea. Pulmonary involvement was presented in half of patients with prevalence on bilateral and upper lobes. The most prevalent sarcoidosis state was state I in $50 \%$ of cases. Conclusion: Although Sarcoidosis it's a rare disease, it represents a challenge in the diagnosis and medical treatment. The series presented are consistent with those reported in other latitudes data.

Key words: Sarcoidosis, pulmonary sarcoidosis, lung diseases.

\section{INTRODUCCIÓN}

La sarcoidosis es una enfermedad sistémica granulomatosa de causa desconocida. Por su connotación sistémica puede afectar la gran mayoría de los órganos corporales; sin embargo, los más afectados son el sistema respiratorio, sistema ocular y la piel. ${ }^{1}$

En Estados Unidos se estima una prevalencia entre el 12 y 23\%,; aunque, los valores de prevalencia varían dependiendo del lugar geográfico, lo que apoya la teoría de la influencia de factores ambientales, genéticos y exposicionales sobre la génesis de la sarcoidosis. ${ }^{2}$ En Colombia se desconoce la prevalencia de la enfermedad.

En algunos estudios se han reportado mayor prevalencia en el sexo femenino, ${ }^{3}$ mientras que en otros no existe diferencia en la presentación de la enfermedad respecto al sexo. ${ }^{4}$ En cuanto a la edad suele presentarse en edades tempranas, siendo rara la aparición de la enfermedad en la niñez o en los adultos mayores. ${ }^{2}$ La raza también se ha visto implicada en los cambios en la prevalencia, ya que hay mayor cantidad de casos en la comunidad afroamericana y caucásica, mientras que en hispanos y asiáticos la prevalencia es menor. ${ }^{5}$

La enfermedad genera altos costos, derivados del tratamiento, hospitalizaciones y ausentismo laboral. Las hospitalizaciones por sarcoidosis son predominantes en los pacientes de edades medias; sin embargo, la mortalidad en las hospitalizaciones aumenta a expensas de los adultos mayores. ${ }^{6}$

Dado que en Colombia no hay suficientes datos para comprender el comportamiento de la enfermedad en la región, el objetivo del presente trabajo es mostrar la experiencia de un centro de referencia del nororiente colombiano, de tal manera que se permita la genera- 
ción de hipótesis y la generación de futuros trabajos de investigación en el tema.

\section{MÉTODOS}

Se realizó revisión de todos los pacientes con diagnóstico clínico de sarcoidosis entre el mes de enero de 2012 hasta el mes de julio del año 2016. Se incluyeron finalmente en la serie de casos, sólo los pacientes que tuvieron confirmación histopatológica de la enfermedad manifestado por la presencia de granulomas no caseificantes con células epiteloides en biopsia de tejido comprometido.

Se diligenciaron los datos de cada paciente en un formato de recolección de datos generado usando el software Epi Info 7.0. Después se exportó la base de datos a formato excel y se analizaron los datos mediante el paquete estadístico Stata 12.0. Se efectuó análisis descriptivo de las variables cuantitativas mediante medidas de tendencia central y dispersión, y de las variables cualitativas mediante frecuencias absolutas y relativas.

\section{RESULTADOS}

En el período evaluado (enero 2012 a julio 2016) se encontró un total de 11 pacientes con diagnóstico clínico de sarcoidosis. De los 11 sujetos preseleccionados, sólo a 8 se les realizó diagnóstico histopatológico de sarcoidosis, por lo que fueron los que finalmente se incluyeron en la serie de casos. La edad media de los sujetos fue de 40 años, con una desviación estándar de 17 años, el $50 \%$ de los pacientes correspondieron al sexo masculino. El resumen de las características de los pacientes se incluye en la tabla 1.

Todos los individuos presentaron síntomas al momento del diagnóstico, siendo el más prevalente la presencia de disnea en el $75 \%$, seguido por tos, pérdida de peso y dolor torácico en el $50 \%$ de los casos. Menos frecuente fue la presencia de fiebre, astenia, expectoración y diaforesis. Los síntomas presentados por los pacientes se muestran en la figura 1.

En cuanto al compromiso respiratorio se encontró en la mitad de los participantes, siento en el total de los casos el compromiso bilateral y de predominio en lóbulos superiores. El compromiso ganglionar mediastinal se determinó en el $87.5 \%$ de los pacientes con sarcoidosis. El estado de sarcoidosis más frecuente fue el estado 1 en el $50 \%$ de los pacientes, seguido por estado $2(25 \%)$ y finalmente 3 y 4 (12.5\%). En la figura 2 se muestra la distribución por estados de la sarcoidosis de los pacientes en la serie de casos.

Se evaluaron variables hematológicas encontrando valor medio de hemoglobina de 13 (desviación estándar de 1.43), media de leucocitos 9,313 (desviación estándar de 4.104) y valor medio de plaquetas de 393.833 (desviación estándar de 152.615). En la tabla 2 se resumen estas variables.

\section{CONCLUSIONES}

La sarcoidosis es una enfermedad poco prevalente en nuestro medio, lo anterior se ve reflejado en que de los cuatro años y medio revisados, sólo se confirmó la presencia de la patología en ocho pacientes.

Como en algunos estudios realizados en otros lugares del mundo, la prevalencia de la enfermedad entre hombres y mujeres fue similar. Si bien el dato no es extrapolable, dado el bajo número de casos analizados, es posible inferir que probablemente en nuestra población la presentación de la enfermedad no tenga diferencias respecto al sexo de los individuos.

En cuanto a la edad se encuentran datos consistentes con los resultados externos, pues se encontró a la mayoría de los pacientes en edad de adulto jovenmedio y sólo dos pacientes llegando a la adultez mayor.

Llama la atención que todos los pacientes se encontraban sintomáticos y, más aún, que los síntomas respiratorios estaban presentes en sujetos

Tabla 1. Características generales de los pacientes con sarcoidosis.

\begin{tabular}{ccccccc}
\hline Código & $\begin{array}{c}\text { Edad } \\
\text { (años) }\end{array}$ & Sexo & $\begin{array}{c}\text { Compromiso } \\
\text { pulmonar }\end{array}$ & Bilateral & $\begin{array}{c}\text { Lóbulos } \\
\text { superiores }\end{array}$ & $\begin{array}{c}\text { Estado } \\
\text { sarcoidosis }\end{array}$ \\
\hline 1 & 25 & Femenino & Presente & Presente & Presente & 2 \\
2 & 34 & Masculino & Ausente & -- & -- & 1 \\
3 & 51 & Femenino & Presente & Presente & Presente & 4 \\
4 & 23 & Masculino & Ausente & -- & -- & 1 \\
5 & 24 & Femenino & Presente & Presente & Presente & 3 \\
6 & 63 & Masculino & Presente & Presente & Presente & 2 \\
7 & 34 & Masculino & Ausente & -- & -- & 1 \\
8 & 64 & Femenino & Ausente & -- & -- & 1 \\
\hline
\end{tabular}




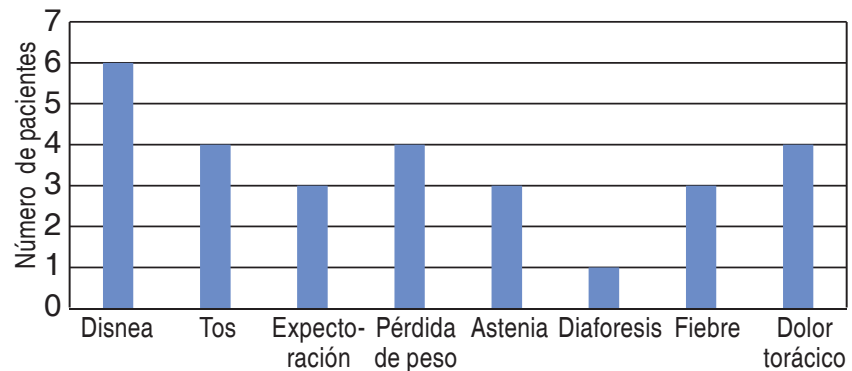

Figura 1. Síntomas de los pacientes con sarcoidosis.

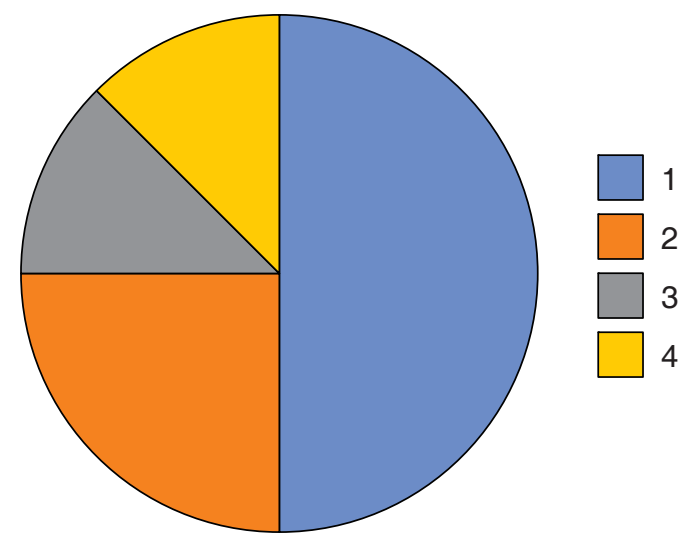

Figura 2. Estados de la sarcoidosis.

Tabla 2. Variables hematológicas pacientes con sarcoidosis.

\begin{tabular}{cccc}
\hline Código & $\begin{array}{c}\text { Hemoglobina } \\
(\mathrm{g} / \mathrm{dL})\end{array}$ & $\begin{array}{c}\text { Leucocitos } \\
\left(\mathrm{cel} / \mathrm{mm}^{3}\right)\end{array}$ & $\begin{array}{c}\text { Plaquetas } \\
\left(\mathrm{cel} / \mathrm{mm}^{3}\right)\end{array}$ \\
\hline 1 & 14.5 & 15.400 & 575 \\
2 & 12.8 & 7.800 & 371 \\
3 & 14.3 & 4.420 & 176 \\
4 & 13.9 & 5.980 & 309 \\
5 & -- & -- & -- \\
6 & 11.4 & 12.400 & 561 \\
7 & -- & -- & -- \\
8 & 11.3 & 9.880 & 371 \\
\hline
\end{tabular}

sin compromiso pulmonar radiológico. Con lo anterior podría llegar a plantearse que existe la posibilidad de compromiso pulmonar no evidenciable mediante imágenes del tórax que sea responsable de la sintomatología de los pacientes, por lo que deberían explorarse otras pruebas diagnósticas, como difusión de monóxido de carbono, para determinar compromiso pulmonar temprano en la sarcoidosis.

Las variables hematológicas no mostraron un patrón característico, por lo que podría concluirse que en la población evaluada, la sarcoidosis podría no estar afectando el sistema hematológico. No obstante esta conclusión no debe generalizarse debido a los pocos pacientes presentes en la serie de casos, pero podría explorarse en estudios con mayor volumen de sujetos.

Finalmente se obtiene otro dato concordante con hallazgos foráneos, en el que el estado de la sarcoidosis más frecuente fue el estado 1 en el $50 \%$ de los casos, siendo el menos frecuente el estado 3 y 4.

\section{REFERENCIAS}

1. Liu D, Birnbaum AD. Update on sarcoidosis. Curr Opin Ophthalmol 2015;26(6): 512-516. doi: 10.1097/ ICU.0000000000000207.

2. Arkema EV, Grunewald J, Kullberg S, Eklund A, Askling J. Sarcoidosis incidence and prevalence : a nationwide register-based assessment in Sweden. Eur Respir J [Internet]. 2016;48(2): 1690-1699. Available from: http:// dx.doi.org/10.1183/13993003.00477-2016

3. Erdal BS, Clymer BD, Yildiz VO, Julian MW, Crouser ED. Unexpectedly high prevalence of sarcoidosis in a representative U.S. Metropolitan population. Respir Med 2012;106(6):893-899. Available from: http://dx.doi. org/10.1016/j.rmed.2012.02.007

4. Thomeer M, Demedts M, Vandeurzen K; VRGT Working Group on Interstitial Lung Diseases. Registration of intersticial ling diseases by 20 centers of respiratory medicine in Flanders. Acta Clin Belg 2001;56(3):163-172.

5. Baughman RP, Field S, Costabel U, et al. Sarcoidosis in America. Analysis based on health care use. Ann Am Thorac Soc 2016;13(8):1244-1252. doi: 10.1513/ AnnalsATS.201511-7600C.

6. Pohle S, Baty F, Brutsche M. In-Hospital Disease burden of sarcoidosis in Switzerland from 2002 to 2012. PLoS One 2016;11(3):e0151940. doi: 10.1371/journal. pone. 0151940.

\section{$\triangle$ Correspondencia:}

Dr. Yeison Santamaría-Alza, Universidad Industrial de Santander-Hospital Universitario de Santander, Bucaramanga, Colombia.

Correo electrónico: yeison-1807@hotmail.es

Los autores declaran no tener conflictos de intereses. 